\title{
Mobile Sink Data Gathering Technique for Wireless Sensor Networks using PSO
}

\author{
Govindarajan Saravanan $^{1 *}$, Madihally JanardhanaIyengar SrinivasaRangachar ${ }^{2}$ \\ ${ }^{I}$ Sri Sairam Institute of Technology, Chennai, India \\ ${ }^{2}$ Hindustan University, Padur, India \\ *Corresponding author's Email: saravanang0671@gmail.com
}

\begin{abstract}
To overcome the challenges of Data gathering and enhancement of lifetime of mobile nodes we propose a new data gathering technique with multiple mobile sinks based on particle swarm optimization (PSO) technique. Particle changes its condition according to the following three principles in the basic particle swarm optimization algorithm: (1) to keep its inertia (2) to change the condition according to its most optimist position (3) to change the condition according to the swarm is most optimist position. We apply PSO technique that finds the current optimal trajectory in the sub-trees for the RP selection. Moreover, by applying the splitting-tree technique, we get the global optimal length constrained trajectory.
\end{abstract}

Keywords: Wireless sensor networks;particle swarm optimization; mobile nodes; Data gathering.

\section{Introduction}

\subsection{Wireless Sensor Networks (WSN)}

Wireless sensor networks (WSNs) consist of many battery-powered tiny sensors. They are capable of mainly two functions namely environmental monitoring, battlefield monitoring, and construction distortion detection. Energy conservation is an important factor in WSNs Wireless sensor networks.It constitutes an upcoming technology that has received a significant attention in the field of industry and academia. Sensor networks can be used for a large range of attractive applications where lifetime of sensor networks and optimized energy consumption is of supreme importance. Moreover, they are selforganizing adhoc systems $[1,2,3]$.

\subsection{Issues of WSN}

The key concern of Sensor nodes is the energy problem. They also have limited resources in terms of computation power, memory, battery power, and transmission capability. In order to reduce energy consumption low power devices can be used

Since data should be transferred from node to node, this causes sensor devices to consume significant amounts of energy due to the execution of a routing protocol. There is also an increase in implementation complexity $[4,5]$.

\subsection{Lifetime of WSN}

System lifetime of a sensor network has many major concerns like maintaining its desired functionality, maintaining enough connectivity, covering entire area, or keeping miss rate below a certain level nodal lifetime, the lifetime of individual sensor nodes is dependent on both given battery capacity and energy consumption rate. The primary challenge for this energy- constrained system is designing energy-efficient protocols to maximize the lifetime of the network, whereas the lifetime of the network is the time at which the first node in the network runs out of energy $[1,6,7]$.

\subsection{Data gathering using Mobile Sinks}

A WSN is generally composed of thousands of sensor nodes, and each sensor is capable of sensing the environment and sending data-to-data observers. Each sensor node is usually equipped with a simple and low-cost computational module due to limited battery lifetime and a low-cost requirement. The collected data is disseminated to a static control 
point, that is, data sink in the network by using node-to-node multi-hop data propagation. Due to the execution of a routing protocol sensor device consumes significant amounts of energy. This improves the implementation complexity. A point of failure arises in the area closer to the control center where the nodes relay the data from other nodes located farther away $[8,9,10]$.

\subsection{Overview}

At first, the mobile sinks (MS) rove around the network to collect all the sensor data by visiting the Rendezvous Points (RPs) [11]. PSO technique finds the current optimal trajectory in the sub-trees, which rooted at one of median's children for the RP selection. We get the global optimal length constrained trajectory by applying the splitting-tree technique [9].The biased sink mobility finds MS mobility with adaptive stop times [12]. The sink stops at each region of RP for an appropriate interval to collect data. The stopping depends on the local node density, data generation rate and the distance between each RPs.

The rest of the paper is arranged as follows: a concise account of certain literary works in Section 2 , a description of proposed method illustrated in Section 3. The test outcomes and relative analysis debate are offered inSection 4. At last, the conclusions are furnished in Section 5.

\section{Literature Review}

W. Liang et al [2] formulated the problem of mixed integer linear programming where the authors devised a heuristic because of its N-P hardness. Based on experiments conducted by simulations, the performance of algorithm is evaluated in terms of network lifetime. Results show that the solution provided by the heuristic is approximately optimal and it is comparable with the one by solving the formulations of mixed integer linear programming. But the running time is short.

To extend the network lifetime C. Hua et al [6] have proposed an optimal routing and data aggregation scheme in WSN.Network lifetime can be prolonged by jointly optimizing data aggregation, routing. A model was adopted to merge data aggregation and the underlying routing scheme, and a smoothingapproximation function was presented for solving the optimization problem. The essential and adequate conditions is derived to achieve the optimality and to design a distributed gradient algorithm. Results also show that the scheme can significantly reduce the data traffic by prolonging the network lifetime
I. Chatzigiannakis et al [8] discussed the fundamental concepts of having a sink, which moves in the network area and collects data from sensor nodes. There are four characteristic mobility patterns for the sink. These patterns are then combined with various data collection strategies. Many important performance properties for each approach are evaluated through simulation. By using sinks mobility and shifting task from sensors to the powerful sink, energy spent can be minimized in relaying traffic, thereby extending the network lifetime.

E. M. Saad et al [3] proposed a well-adaptive moving strategy for a mobile sink in large-scale, hierarchical WSN. In scenarios where there is time constraints, the mobile sink traverses the whole network to upload the sensed data from cluster heads. The authors design the mobile sink trajectory in such a way that all cluster heads do not need multi-hop relays to reach the mobile sink. Thus, the network lifetime is extended for attaining high level of energy efficiency and for a fair balancing of the energy consumption across all network cluster heads

M. Ma et al [9] have proposed a problem for planning the moving path of a mobile observer to balance the traffic load from the sensors to the mobile observer. Thereby network lifetime could be prolonged. Several applications such as environment monitoring for some human-unreachable environmentwas considered. For instance, outer space, the seabed, and so on where the sensed data is generally collected at a low rate and the sensed data is not susceptible to time delayfor it to be accumulated into fixed-length data packets and uploaded once in a while.

M. Hossein et al [13] have proposed an energyefficient Network data aggregation approach in WSN, which uses the advantages of both cluster, based and tree based approaches. The energy consumption in wireless transmissions is equivalent to the square of distance between two nodes in communication. In this approach, data is transmitted by the nodes to their neighbor instead of their cluster head. Therefore, there is a reduction in communication distance and the energy consumption of each node. Thereby each cluster and the average energy consumption of the whole networks is reduced and the network lifetime is increased

By adopting mobility and space-division multiple accesses (SDMA) technique to optimize system performanceM. Zhao et al in paper [14] addressed the data gathering issue in WSNs. A mobile data collector, for convenience, called SenCar in this paper is deployed in a WSN.The data 
is sent to the SenCar by the sensor without any relay so that the lifetime of sensors can be prolonged. Application of SDMA technique is considered to data gathering by equipping the SenCar with two antennas. The problem of minimizing the total time of a data gathering tour, which consists of two parts: data uploading time and moving time is focused on.

J. Du et al [11] have proposed ME in heterogeneous WSN where there is a distinct data generation rates of sensors. This is contrary to the previous works where the authors assumed that the sensory data couldn't be aggregated and the link quality instable. These assumptions highlight the dynamic nature of WSNs; which was more practical. On gathering the global data, the energy consumption is optimized. ME can efficiently collect network-wide data within a given period. In order to prolong its lifetime, the network reduces the energy bottleneck.

$\mathrm{X}$. Wu et al [15] have proposed Dual-Sink which is an energy-efficient and distributed protocol for data collection, Since a mobile sink and a static sink are used, the mobile sink broadcasts within a limited range instead of network throughout.

Z. Sha et al [16] have proposed a moving strategy for the mobile sink .where adversaries cannot track or detect this strategy during its data collection phase around the sensor environment.A trajectory is selected for mobile sink node to reduce the total number of message communication from all static sensor nodes to the mobile sink node. .This includes multihop relaying, which reduces the possibility of being detected by the adversaries. To forward the data to mobile sink node in the shortest path a routing protocol is used on sensor nodes

With respect to energy and latency in WSNA. Kinalis et al [12] have proposed biased sink mobility with adaptive stop times for efficiently collecting the data. A weak model of a single mobile sink is assumed, where a strategy for network traversal has been proposed .This assists the nodes in a balanced way. Traversal is done on a per region basis .where regions are visited by the sink one after another. At each region, sink waits for an appropriate interval to gather data.

In heterogeneous WSN, the authors have studied the data collection problem for the mobile element where there is a distinct data generation rates of sensors.ME can efficiently gather network-wide data within a specific period.

In [12] they have proposed biased sink mobility with adaptive stop times, for efficient (with respect to both energy and latency) data collection in wireless sensor networks. However, in this approach the sink mobility is high and is not minimized. At the same time, the network removes the energy bottleneck in order to extend the network lifetime. In this approach, the energy consumption is optimized on collecting the global data.

As a solution to the problems of the existing works a new data gathering technique with multiple mobile sinks based on particle swarm optimization (PSO) technique is proposed

PSO has been used by many applications for a series of problems. The algorithm of PSO emulates from behavior of animals from societies that do not have any leader in their group or swarm, such as bird flocking and fish schooling. Typically, a flock of animals that have no leaders finds food by random, follows one of the members of the group that has the closest position with a food source (potential solution). Through communication among members who already have a better situation that is attained by the flocks. An animal, which has a better condition, is informed it to its flocks and the others moves simultaneously to that place. This happens as a continuous process until the best conditions or a food source is discovered.The process of PSO algorithm in finding optimal values follows the work of this animal society where PSO consists of a swarm of particles. Here a particle represents a potential solution [17].

\section{Overview}

In particle swarm optimization,each particle occupies a space in D-dimensional and changes its condition according to the following three principles:

(1) To keep its inertia (2) to change the condition according to its most optimist position (3) to change the condition according to the swarm's most optimist position.The position of each particleinfluenced by velocity depends upon the optimist position during its movement (individual experience) and the position of the most optimist particle in its surrounding (near experience).

\subsection{Particle Swarm Optimization}

In particle swarm optimization, particle swarm consists of " $n$ " particles each particle maintains its position based on global best fit and candidate best fit. Candidate best fit is the best position and global best fit is the knowledge of the global or their neighborhood's best.

Let $P_{i}(t)$ denotes the position of particle $i$ at time t. ' $i$ ' is the index of the particle and $v_{i}(t)$ is the velocity of particle $i$ at time $t$. Then the velocity $\left(V_{i}\right)$ of the particle is calculated using the following equation [17] 


$$
\begin{aligned}
& V_{i}(t+1)=V_{i}(t)+c_{1} r_{1}\left[\left(C B F(t)-P_{i}(t)\right]+\right. \\
& c_{2} r_{2}\left[\left(G B F(t)-P_{i}(t)\right]\right.
\end{aligned}
$$

In the equation (1), $\mathrm{CBF}$ is the candidate best fit and GBF is the global best fit. $c_{l}, c 2$ are user supplied coefficients and $r_{1}, r_{2}$ are random values generated for each velocity update.

The position of the particle changes by adding the velocity $\left(V_{i}\right)$ to the current position and we calculate the new position of particle using the following equation [17]

$$
P_{i}(t+1)=P_{i}(t)+V_{i}(t+1)
$$

\subsubsection{Intertia Component $\left(\mathrm{WV}_{\mathrm{i}}\right)$}

Intertia component helps the particle to move in the same direction and its typical value ranges between 0.8 and 1.2. Lower values of the inertial coefficient speed up the convergence of the swarm to optima, and higher values of the inertial coefficient encourage exploration of the entire search space.

\subsubsection{Optimist position: Optimistposition}

Changes the condition according to its most optimist position. It takes all the candidate best fit values and finds the optimist position. It finds the most optimist position among the candidate best-fit values. High individual candidate fit value affects the size of the each particle.

\subsubsection{Swarm's most Optimist Position}

Swarm's most optimist position depends upon the global best-fit value. The swarm's most optimist position depends upon the swarm movement. The swarm's optimist position calculation is .based on the each swarm global fit value.

\subsection{Splitting - Tree}

Consider the node as N. The optimal trajectory lays in one of the sub-trees by removing the node $\mathrm{N}$ from the tree and passes through node for a specific node.Recursivealgorithmfinds the global optimal length constrained trajectory.

Initially, the algorithm begins with the calculation of AVG values of the nodes. It is based on the candidate best-fit value. Classification of tree occurs based on these AVG value into number of sub-trees. The sub-tree goes on dividing into subtrees, until it gets the optimal length. If the node does not get any optimal length value, it will restore the optimal trajectories' candidates into the candidate set. The following diagram represents the Splitting tree.
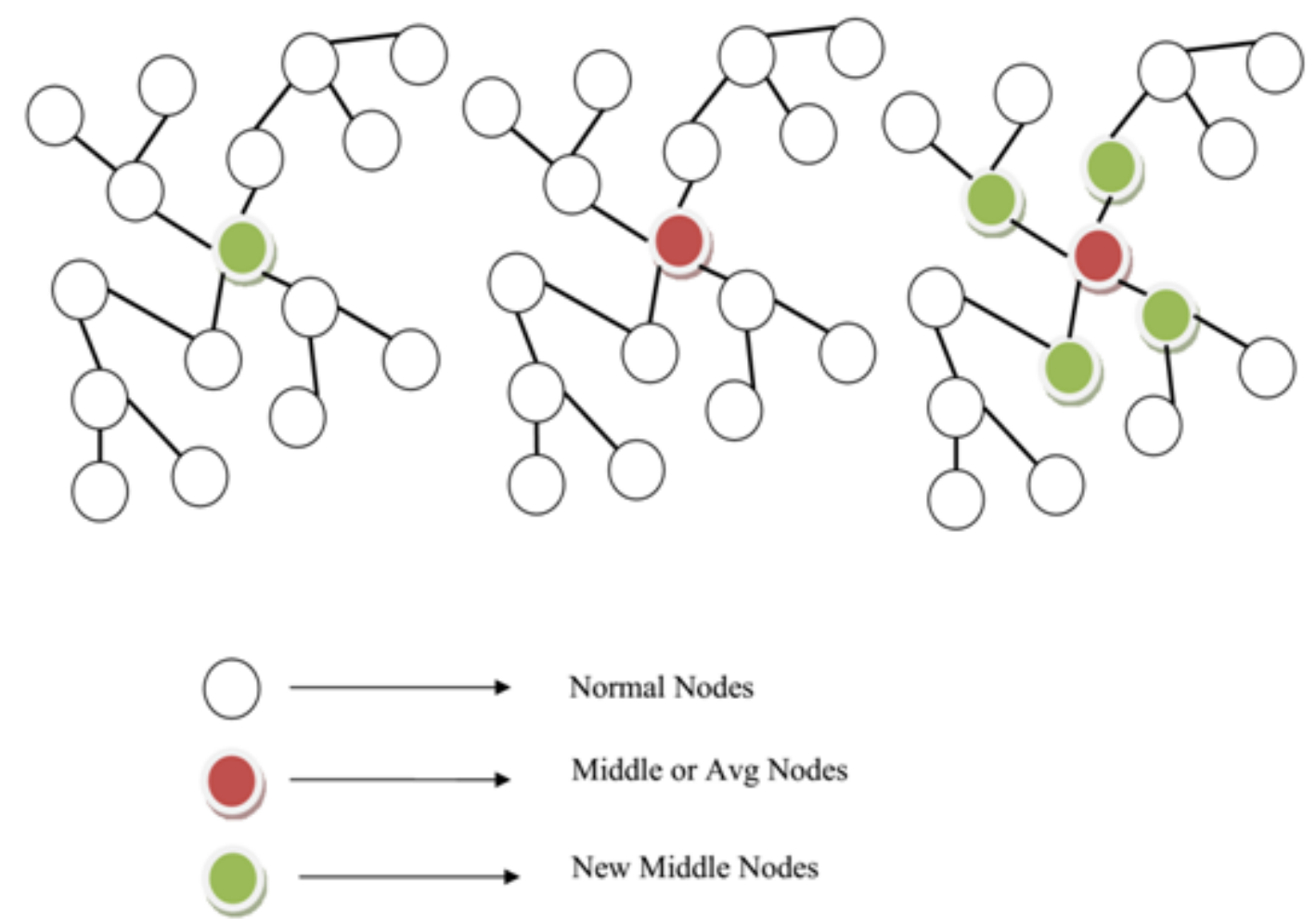

Figure.1 Splitting tree 
The above example explains the splitting tree concept. Initially, the tree contains the normal nodes. Selection of middle node depends on the average value. After calculating the middle node, the tree undergoessub classificationusing fitness function.

$$
\text { Fitness Function }\left(F_{t}\right)=[\alpha * S(T)-L(T)]
$$

In the equation (3), $S(T)$ is the size of the tree and $L(T)$ is the number of leaf nodes in the tree. $\alpha$ is the random number. Based on this fitness function, we split each sub-tree. Each sub-tree contains one middle node.

1. Start

2. Define Avg $=$ average value ( $\mathrm{m})$,

$\mathrm{q}=$ Queue,

$\mathrm{S}=$ Candidate Set;

3. Find the $m$ value of the tree-shaped topology

4. root the m nodes as the tree-shaped topology node

5. push $m$ into the $q$

6. while $\mathrm{q} !=$ NULL do

7. find the optimal length

8. Insert $\mathrm{P}_{\mathrm{o}}$ value into the $\mathrm{S}$

9. Insert the children of $\mathrm{N}$ nodes $\mathrm{q}$

10. End While

11. Find the Global optimal length with the minimum optimal length of the each $\mathrm{P}_{\mathrm{o}}$.

12. Return the minimum $\mathrm{P}_{\mathrm{o}}$.

\section{Algorithm 1: Splitting - Tree}

Algorithm (1) is the splitting tree algorithmthatexplains calculation process inthemiddle nodes and the division of tree into the sub trees. PSO technique selects RP by finding the current optimal trajectory in the sub-trees, which is rooted at one of median's children.

In splitting tree, we use the PSO technique.

1. Start

2. Define $\mathrm{T}=$ Full Tree,

$\mathrm{T}_{\mathrm{i}}=$ Sub Tree.

3. If ( end of the (T))

4. \{

5. Split the tree using the fitness function $\left(\mathrm{T}_{\mathrm{i}}\right)$

6. Calculate the inertia of each node in the sub tree $\left(\mathrm{T}_{\mathrm{i}}\right)$

7. Calculate each node optimist position in the sub tree $\left(\mathrm{T}_{\mathrm{i}}\right)$

8. Calculate entire tree optimist position in the sub tree $\left(\mathrm{T}_{\mathrm{i}}\right)$
9. Rearrange the tree based on the inertia, node optimist position and tree optimist position.

10. \}

11. End

\section{Algorithm 2: Splitting Tree using the PSO}

In algorithm (2), the tree divides into sub tree using the fitness function. Each node in the sub tree needs to calculate inertia, each node optimist position and tree optimist. After calculating these three values, the sub tree arranges into new form. Then by applying the splitting-tree technique, we get the global optimal length constrained trajectory.

\subsection{Adaptive Stop Times}

The sensor changes very frequently in order to communicate with the sink node for updating it changes. For collecting all the recorded data, the sink needs to visit each sensor several times. Since the generation of data occurs continuously, none of the data can be collected in a single session with a sensor.

For this problem, we use biased sink mobility with adaptive stop times. Sink traverses the graph G, for a certain amount of time and adaptive stop time. The amount of time is constant. Adaptive stop time depends upon the local parameters.

Each case contains stopping times. Variable total stop time is the totally amount of time the sink can remain inactively and is represented as $T_{s}$ It is calculated using the following equation

$$
\begin{aligned}
\mathrm{TIE} & =\text { total messges sent } * \mathrm{E}_{\text {msg }} \\
& =\mathrm{n} * \mathrm{G} * T_{S} * \mathrm{E}_{\text {ms g }}+\mathrm{E}_{\mathrm{idle}}
\end{aligned}
$$

In equation (4), TIE is the total initial energy of all the sensors in the network, where $\mathrm{n}$ is the number of sensors of the network and $\mathrm{G}$ is the average event generation rate. We calculate the maximum stop times of the sink value until the whole energy becomes empty.

\subsection{Example for Splitting - Tree using PSO}

In the above figure (2), 2 (a) is the indicial tree and it do not have any middle nodes. Drepresents Middle node. All the nodes are in a balanced manner. The nodes point towards the middle node creating an optimal path. The middle node splits the tree into sub trees. The path is $\mathrm{E}->\mathrm{M}->\mathrm{F}$. 




(a) Indicial tree

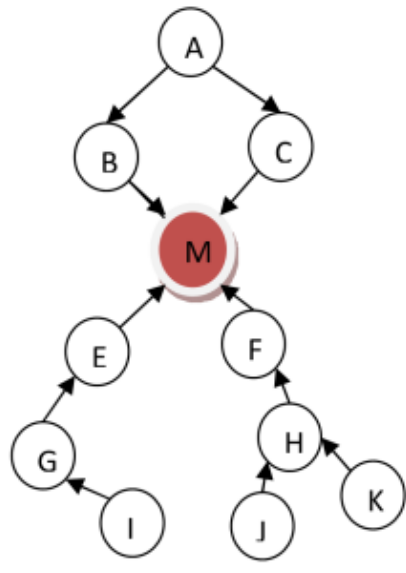

(b) Sub-tree



(c) Tree optimist

Figure.2 splitting tree Example

\section{Advantages}

- Collection of global data optimizes the energy consumption

- The mobile sink prolongs the lifetime of the network

- The mobile sink collects the data effectively with adaptive stop times

- The moving strategy minimizes the total number of message communication of the mobile sink

- The Mobile Element collects network-wide data efficiently within a given delay bound

\subsection{Total Work Flow}

The stopping time of mobile sinks, (MS) depends on the local node density, data generation rate and the distance between each RPs. Below shows the work flow chart of the mobile sinks.

1. Start

2. Get the updated sink information using the Adaptive stop times

3. Each sink visits Rendezvous Points (RPs)

4. Form a tree using the updated information

5. Split the tree into sub tree using fitness function

6. Apply the Splitting Tree using the PSO on each sub tree

7. Repeat the process according to the Adaptive stop timer

8. End

\section{Algorithm 3: Total Work Flow}

Using the updated information, PSO technique finds the current optimal trajectory in the sub-trees. There by, allowing the reconstruction of an entire tree.

\section{Simulation Results}

\subsection{Simulation Model and Parameters}

The proposed architecture simulates through Network Simulator (NS2) [18]. During the simulation process, the mobile nodes move in a 500 meter $\times 500$ meter region for 50 seconds of simulation time. All nodes have the same transmission range of 250 meters. The simulated traffic is also known as Constant Bit Rate (CBR).

Below table summarizes the simulation settings and parameters

Table 1. Simulation parameters

\begin{tabular}{|c|c|}
\hline No. of Nodes & $20,40,60,80$ and 100 \\
\hline Area Size & $500 \times 500$ \\
\hline Mac & IEEE 802.10 \\
\hline $\begin{array}{c}\text { Transmission } \\
\text { Range }\end{array}$ & $250 \mathrm{~m}$ \\
\hline Simulation Time & $50 \mathrm{sec}$ \\
\hline Traffic Source & CBR \\
\hline Routing Protocol & PSOMSDG \\
\hline Initial Energy & 15.1 \\
\hline $\begin{array}{c}\text { Transmission } \\
\text { Power }\end{array}$ & 0.660 \\
\hline Receiving Power & 0.395 \\
\hline Rate & $\begin{array}{c}50,100,150,200 \text { and } \\
250 \mathrm{~Kb}\end{array}$ \\
\hline
\end{tabular}

\subsection{Performance Metrics}

Below given figures shows, the comparison of the proposed PSO based Mobile Sink Data Gathering Technique (PSO-MSDG) and the Biased Sink Mobility with Adaptive Stop Times (BSMASD) technique [12]. The following metrics evaluates the performance rate.

- Packet Drop: It refers the average number of packets dropped during the transmission 
- Energy Consumption: It is the amount of energy consumed by the nodes to transmit the data packets to the receiver.

- Delay: It is the amount of time taken by the nodes to transmit the data packets.

- Packet Delivery Ratio: It is the ratio between the number of packets received and the number of packets sent.

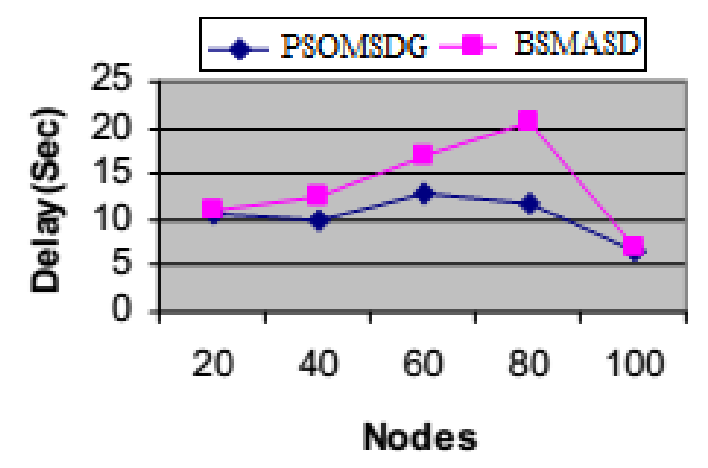

Figure.3 Nodes Vs Delay

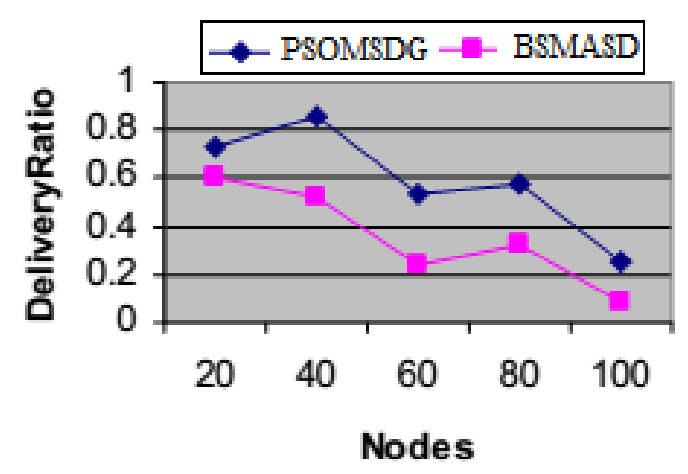

Figure.4 Nodes Vs Delivery Ratio

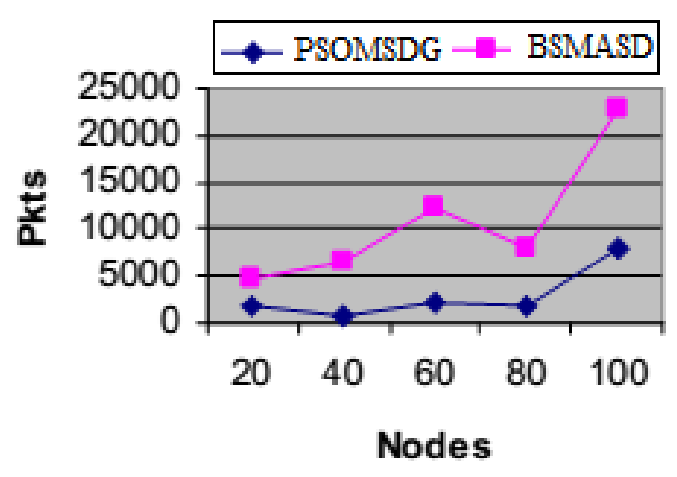

Figure.5 Nodes Vs Drop



Figure.6 Nodes Vs Energy Consumption

\subsection{Results}

\section{A. Based on Nodes}

In our first experiment, we vary the number of nodes as 20,40,60,80 and 100.Figure 3 shows the delay of PSOMSDG and BSMASD techniques for different number of nodes scenario. PSOMSDG shows delay, which is $20 \%$ of less than BSMASD approach.

Figure 4 shows the delivery ratio of PSOMSDG and BSMASD techniques for different number of nodes scenario. The delivery ratio of our proposed PSOMSDG is $44 \%$ higher than BSMASD approach.

Figure 5 shows the drop of PSOMSDG and BSMASD techniques for different number of nodes scenario. The drop of our proposed PSOMSDG is $75 \%$ less than BSMASD approach.

Figure 6 shows the energy consumption of PSOMSDG and BSMASD techniques for different number of nodes scenario. The energy consumption of our proposed PSOMSDG is $8 \%$ less than BSMASD approach.

\section{B. Based on Rate}

In our second experiment, we vary the transmission rate as 50,100,150,200 and $250 \mathrm{~Kb}$.

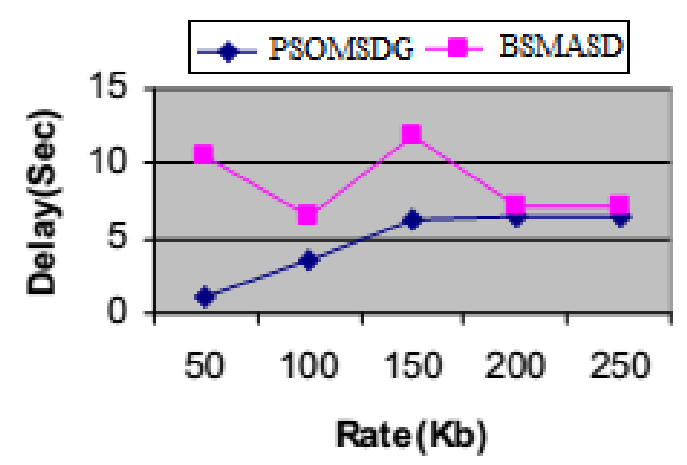

Figure.7 Rate Vs Delay 


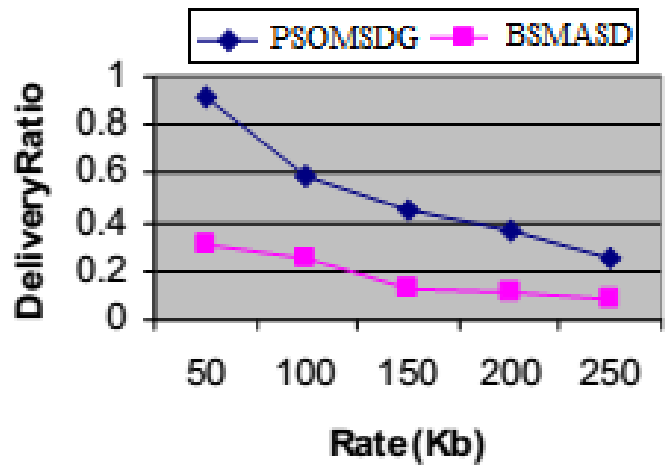

Figure.8 Rate Vs Delivery Ratio

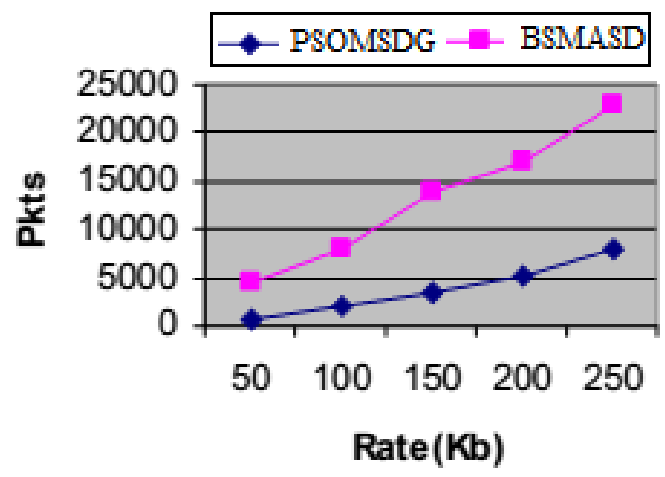

Figure.9 Rate Vs Drop



Figure.10 Rate Vs Energy Consumption

Figure 7 shows the delay of PSOMSDG and BSMASD techniques for different rate scenario. The delay of our proposed PSOMSDG is $40 \%$ less than BSMASD approach.

Figure 8 shows the delivery ratio of PSOMSDG and BSMASD techniques for different rate scenario. The delivery ratio of our proposed PSOMSDG is $66 \%$ higher than BSMASD approach.

Figure 9 shows the drop of PSOMSDG and BSMASD techniques for different rate scenario. The drop of our proposed PSOMSDG is $73 \%$ less than BSMASD approach.

Figure 10 shows the energy consumption of PSOMSDG and BSMASD techniques for different rate scenario. The energy consumption of our proposed PSOMSDG is 7\% less than BSMASD approach.

\section{Conclusion}

This paper proposes a new data gathering technique with multiple mobile sinks based on particle swarm optimization (PSO) technique. In the PSO technique, particles change its condition according to the following three principles: (1) to keep its inertia (2) to change the condition according to its most optimist position (3) to change the condition according to the swarm's most optimist position. For the RP selection, we apply PSO technique, which finds the current optimal trajectory in the sub-trees. Then by applying the splitting-tree technique, we get the global optimal length constrained trajectory. In this approach, all the nodes are in a balanced manner. The sink visits each RPs one after another, stopping at each region for an appropriate interval to collect data. Estimation of stopping depends based on the local node density, data generation rate and the distance between each RPs.Thus, the performance evaluation metrics given output are satisfactory but in order to improve the system efficiency more, as in future work we have planned to propose adaptive optimization algorithm and so that we can improve the efficiency of the proposed system

\section{References}

[1] J. Li and P. Mohapatra, "Analytical Modeling and Mitigation Techniques for the Energy Hole Problem in Sensor Networks", Pervasive and Mobile Computing, Vol. 3, No. 3, pp. 233-254, 2007.

[2] W. Liang, J. Luo and X. Xu, "Prolonging Network Lifetime via A Controlled Mobile Sink in Wireless Sensor Networks", IEEE Communications Society subject matter experts for publication in the IEEE Globecom 2010 proceedings, pp. 1-6, 2010.

[3] E. M. Saad, M. H. Awadalla, M. A. Saleh, H. Keshk, and R. R. Darwish, "A data gathering algorithm for a mobile sink in largescale sensor networks", In Wireless and Mobile Communications, The Fourth International Conference on IEEE, pp. 207-213, 2008.

[4] A. Rajeswari, and R. Manavalan, "Data Collection Methods in Wireless Sensor Network", International Journal for Research in Applied Science and Engineering Technology, Vol. 2, No. 9, pp. 259-272, 2014.

[5] Y. Yao, Q. Cao and A. V. Vasilakos, "An EnergyEfficient, Delay-Aware, and Lifetime-Balancing Data Collection Protocol for Heterogeneous Wireless Sensor Networks", IEEE Transactions on Networking, Vol. 23, No. 3, pp. 810-823, 2015.

[6] C. Hua and T. S. P. Yum, "Optimal Routing and Data Aggregation for Maximizing Lifetime of Wireless 
Sensor Networks", IEEE/ACM Transactions on Networking, Vol. 16, No. 4, pp. 892-903, August 2008.

[7] D. Suresh and K. Selvakumar, "Improving Network Lifetime and Reducing Energy Consumption in Wireless Sensor Networks", Proc. IEEE Transactions on, International Journal of Computer Science and Information Technologies, Vol. 5, No. 2, pp. 1035-1038, 2014.

[8] I. Chatzigiannakis, A. Kinalis and S. Nikoletseas, "Efficient data propagation strategies in wireless sensor networks using a single mobile sink", Vol. 31, No. 5, pp. 896-914, 2007.

[9] M. Ma and Y. Yang, "SenCar: An Energy-Efficient Data Gathering Mechanism for Large-Scale Multihop Sensor Networks", IEEE Transactions on Parallel And Distributed Systems, Vol. 18, No. 10, pp. 14761488, October 2007.

[10] J. Zhang, Z. Yang, B. Wang, and H. S. X Sun, "ECTP: An Energy-balanced collection Tree Protocol for Power Constrained Wireless Sensor Networks", Proc. IEEE Transactions on, International Journal of Grid and Distributed Computing, Vol. 7, No. 2, pp. 115-126, 2014.

[11] J. Du, H. Liu, L. Shangguan, L. Mai, K. Wang and S. Li, "Rendezvous Data Collection Using a Mobile Element in Heterogeneous Sensor Networks", International Journal of Distributed Sensor Networks, 2012.
[12] A. Kinalis, S. Nikoletseas, D. Patroumpa and J. Rolim, "Biased Sink Mobility with Adaptive Stop Times for Low Latency Data Collection in Sensor Networks", Information fusion, Vol. 15, pp. 56-63, 2009.

[13] M. H. Anisi, A. H. Abdullah, and S. A. Razak, "Energy-Efficient Data Collection in Wireless Sensor Networks", Wireless Sensor Network, Vol. 3, No. 10 , pp. 329-333, 2011.

[14] M. Zhao, M. Ma and Y. Yang, "Mobile Data Gathering with Space-Division Multiple Access in Wireless Sensor Networks", IEEE Communications Society subject matter experts for publication in the IEEE INFOCOM 2008 proceedings, pp.1957-1965, 2008.

[15] X. Wu and G. Chen, "Dual-Sink: Using Mobile and Static Sinks for Lifetime Improvement in Wireless Sensor Networks", IEEE, pp. 1297-1302, 2007.

[16] Z. Sha, J.L. Lu, X. Li and M.Y. Wu, "An AntiDetection Moving Strategy for Mobile Sink", IEEE Communications Society subject matter experts for publication in the IEEE Globecom proceedings, pp. 1-5, 2010.

[17] Dian, P. Rini, M. S. Siti, and S. Y. Siti, "Particle Swarm Optimization: Technique, System and Challenges", International Journal of Computer Applications, Vol. 14, No. 1, pp. 19-27, 2011.

[18] Network Simulator: http:///www.isi.edu/nsnam/ns 